\title{
A type 2 papillary renal cell carcinoma presenting as an intracystic necrotic lesion: A case report
}

\author{
ZHENYU FU ${ }^{1}$, LIGUO SUN $^{1}$, YUHUA HUANG ${ }^{2}$, JIE ZHANG $^{1}$, ZICHAO ZHANG $^{3}$, \\ LIJUN WANG $^{4}$, SHENGYU WANG $^{5}$ and GE ZHANG ${ }^{1}$ \\ ${ }^{1}$ Department of Urology, The No. 2 People's Hospital of Changshu, Suzhou, Jiangsu 215500; \\ ${ }^{2}$ Department of Urology, The First Affiliated Hospital of Soochow University, Suzhou, Jiangsu 215006, P.R. China; \\ ${ }^{3}$ Institute of Biosciences and Technology, Texas A\&M Health Science Center, Houston, TX 77030, USA; \\ Departments of ${ }^{4}$ Orthopaedics and ${ }^{5}$ Radiography, The No. 2 People's Hospital of Changshu, \\ Suzhou, Jiangsu 215500, P.R. China
}

Received October 18,2012; Accepted December 3, 2012

DOI: $10.3892 / \mathrm{mco} .2013 .64$

\begin{abstract}
Papillary renal carcinoma (papillary RCC) is a histological subtype of the renal carcinoma, which in turn, has two morphological subtypes that correlate with prognosis. The present study reported an unexpected finding of type 2 papillary renal cell carcinoma (papillary RCC) presenting intracystic necrosis cavity. A cystic renal lesion was identified incidentally in a 66-year-old man during an abdominal computed tomography (CT) scan performed for the evaluation of a gastrointestinal stromal tumor. Subsequent contrast material-enhanced CT scan and magnetic resonance imaging (MRI) examination labeled the mass as category III degree on the basis of the Bosniak classification scheme. Surgical exploration by laparoscopic radical nephrectomy was performed to determine the diagnosis. Definitive pathological study confirmed a type 2 papillary RCC with an intracystic necrosis cavity. To the best of our knowledge, this case demonstrated for the first time a cavity within a papillary RCC, supporting the hypothesis that type 2 papillary RCC could develop cavity avascular necrosis during its cystic degeneration.
\end{abstract}

\section{Introduction}

Papillary renal carcinoma (papillary RCC) is a histological subtype of the renal carcinoma. There are two morphological subtypes of papillary RCC that are correlated with their prognosis. Type 1 tumor consists of papillae enclosed with a single layer of small cells with scanty cytoplasm and low-grade nuclei. In type 2 tumor, the cells covering the papillae are pseudostratified, generally have eosinophilic cytoplasm and

Correspondence to: Dr Ge Zhang, Department of Urology, The No. 2 People's Hospital of Changshu, Suzhou, Jiangsu 215500, P.R. China

E-mail: explorer_dl@hotmail.com

Key words: type 2 papillary renal cell carcinoma, necrosis, cavitation are usually of higher nuclear grade compared to the cells of type 1 tumors. Papillary RCC is usually associated with a more favorable prognosis compared to the carcinoma of renal clear cells, while type 2 tumors carry a worse prognosis compared to type 1 tumors. At the cytogenetic level, the most common karyotypic changes in papillary RCC are trisomy of chromosomes 7 and 17, as well as loss of chromosome $\mathrm{Y}$ in males (1-3). Microscopically, papillary RCCs were predominantly papillary or tubulopapillary, often with foam cells, necrosis, hemorrhage and multifocality (4). This case report described an incidental finding of a necrotic cavity within a papillary RCC. The imaging data documented the dynamic progress of the cavitation within the cystic mass. This study was approved by the Institutional Review Board of the No. 2 People's Hospital of Changshu. Informed consent was obtained from the patient.

\section{Case report}

A 66-year-old male patient underwent a second abdominal computed tomography (CT) scan while being monitored for a renal cyst at the upper pole of the left kidney that was inadvertently detected 22 months earlier in another hospital. The patient's medical history showed surgery had been performed for gastric adenocarcinoma in January, 2010 at the same institution. Pre-operative abdominal CT in 2010 detected an unexpected complex renal cyst at the upper pole of the left kidney (Fig. 1A-C), but no relevant treatment was administered. Following admission, the patient underwent a contrast material-enhanced $\mathrm{CT}$ and magnetic resonance imaging (MRI) examination to evaluate the origin of the mass, its correlation with surrounding structures and possible evidence of metastatic involvement. CT scans demonstrated a $5.7 \times 5.4 \mathrm{~cm}$ well-circumscribed, exophytic mass arising from the upper pole of the left kidney. A distinct lesion of $1.8 \mathrm{~cm}$ at its greatest diameter within the exophytic mass was also detected, with a CT value between -600 to-970 HU (Fig. 1D-F). MRI results showed that the mass exhibited a pseudocapsule and had low signal intensity on T1- and T2-weighted images (Fig. 2). This cystic renal lesion was confirmed as category III 
A

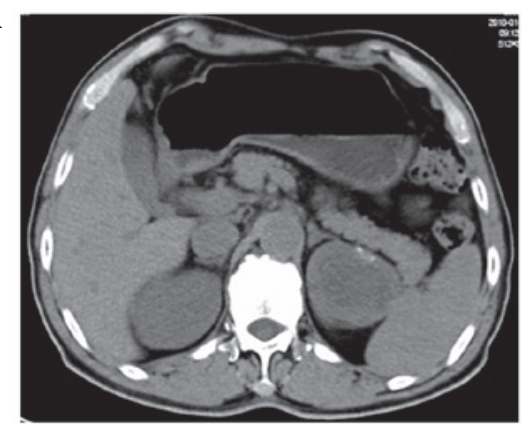

D

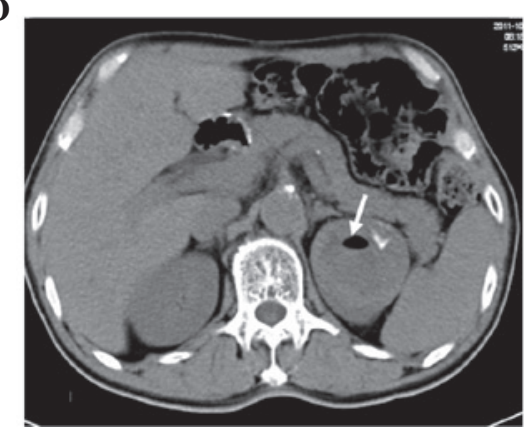

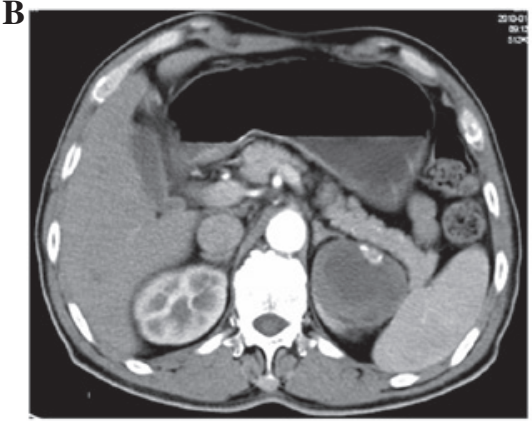

E

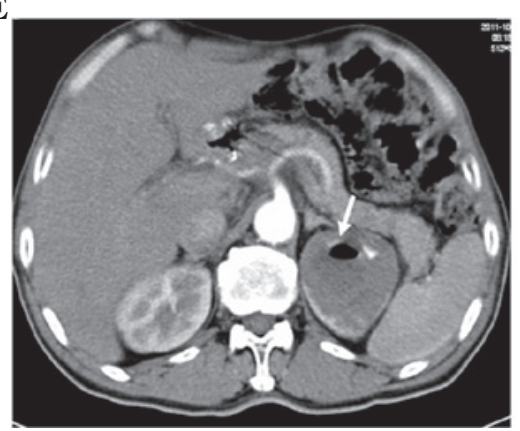

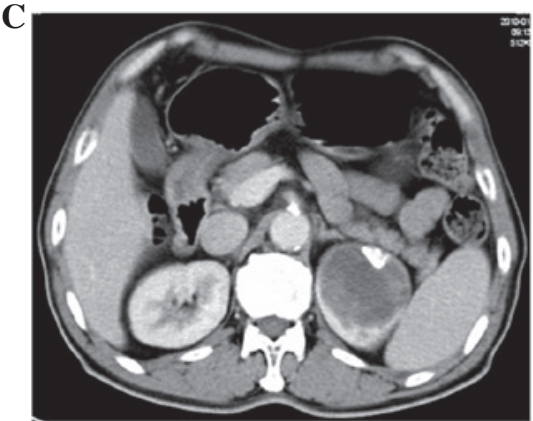

F

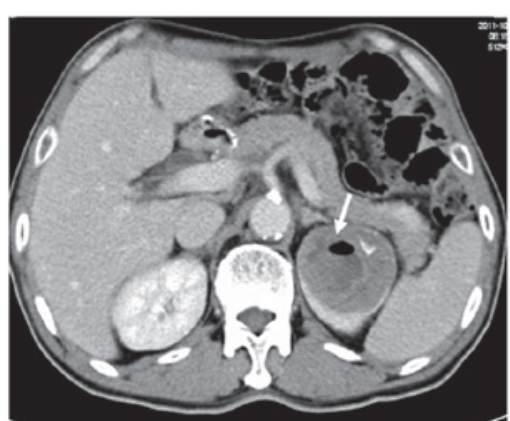

Figure 1. (A) Unenhanced, (B) corticomedullary phase and (C) nephrographic phase CT scans showing a 5.2x4.4-cm low-attenuation mass in the left renal cortex, with extensive necrotic areas, calcification and peripheral enhancement. (D-F) CT scans 22 months later showed a distinct lesion with a maximum diameter of $1.8 \mathrm{~cm}$ within the exophytic renal mass (arrow), with a suspected air CT value between -600 and $-970 \mathrm{HU}$.
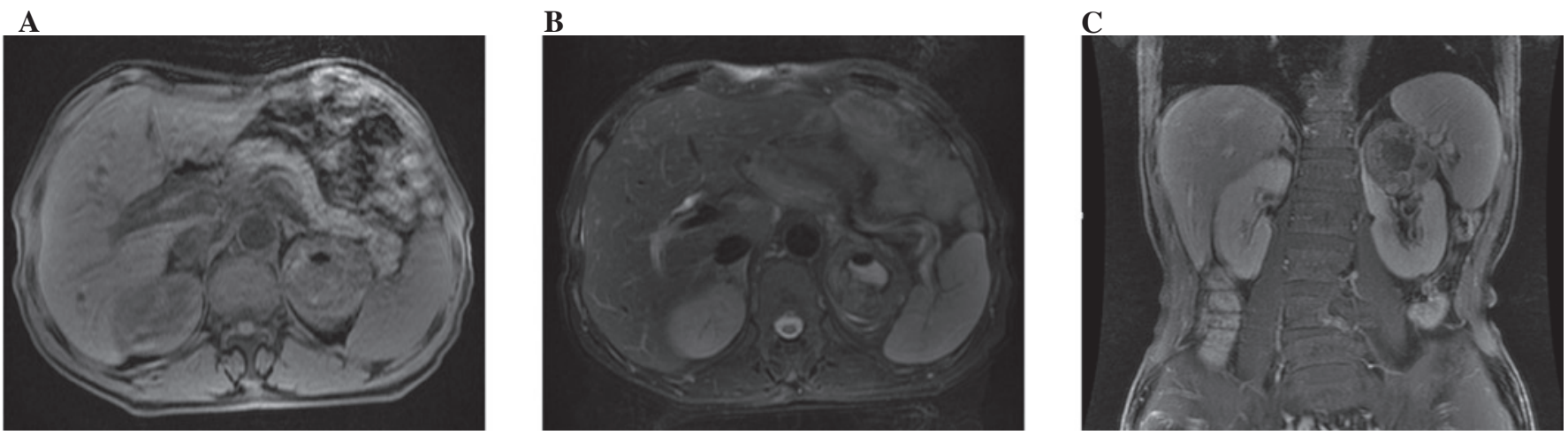

Figure 2. Magnetic resonance imaging (MRI) shows the renal cystic mass exhibiting a pseudocapsule with low signal intensity on T1- and T2-weighted images.

A

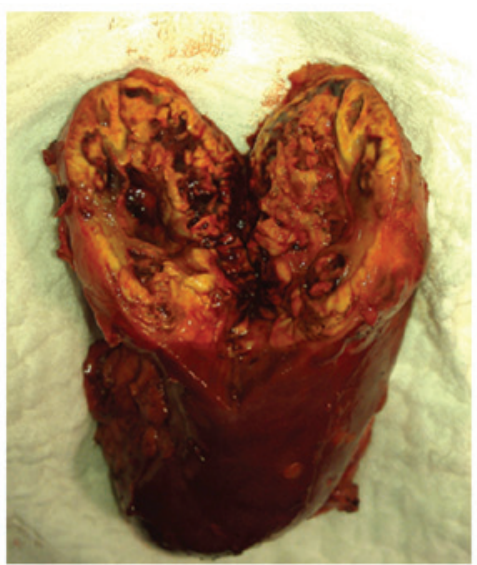

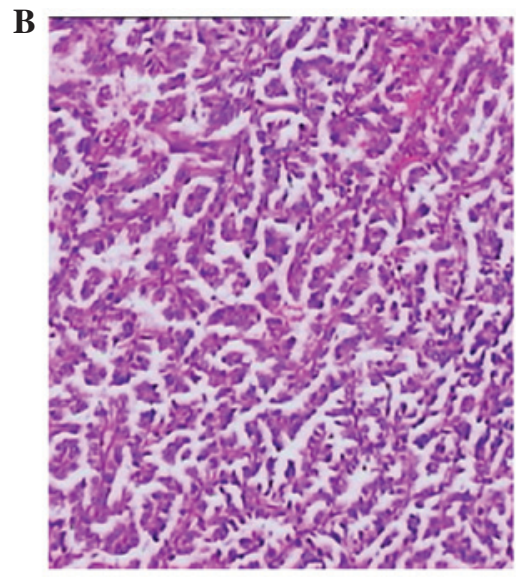

$\mathrm{C}$

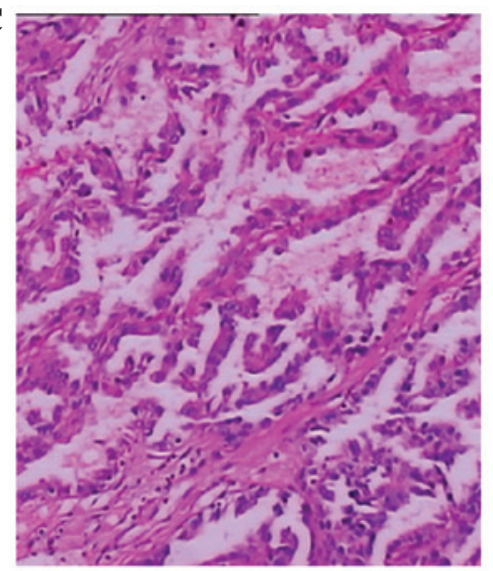

Figure 3. (A) Image of the cut surface of the specimen from radical nephrectomy shows the heterogeneous tumor with a large cavity component secondary to necrosis. (B-C) Histological analysis: representative photomicrographs of tumor sections (original magnification, x100 and x400, respectively; hematoxylin and eosin-stained), showing fibrovascular papillae lined by pseudostratified, large pleomorphic cells with eosinophilic cytoplasm and large, prominent nucleoli. 
on the basis of the Bosniak classification scheme. The patient analysis did not show any abnormalities. Concerning current diagnostic criteria, probable diagnosis for such a heterogeneous cystic mass was limited to a hemorrhagic renal cyst or cell carcinoma.

Since renal carcinoma involves a potential risk of metastasis, radical nephrectomy was scheduled for removal of the left kidney. After general anesthesia was induced, the patient was placed in the lateral recumbent position and laparoscopic left radical nephrectomy was successfully performed in October, 2011. Gross examination was characterized by an irregular-shaped lesion of mahogany-brown color in the upper pole of the left kidney, with a maximum diameter of $5.7 \mathrm{~cm}$. The central area in the exophytic lesion was identified as a $1.8-\mathrm{cm}$ circumscribed cavity, with no liquid inside but with viscous, chocolate-brown, necrotic and hemorrhagic material on the cavity wall. The harvested specimen was processed according to standard surgical pathology protocols. Microscopic examination results (Fig. 3) showed that the specimen exhibited an outer cystic mass in the papillary architecture with pleomorphic cells showing prominent nucleoli and abundant eosinophilic cytoplasm. Papillary interstitial structure comprises cells arranged on a delicate fibrovascular core. Infiltration of foamy macrophages into the papillary structures and extensive tumor necrosis with abundant hemosiderin granules were detected. Immunohistochemical analyses showed the tumor cells to be strongly positive for vimentin, epithelial membrane antigen (EMA) and cytokeratins 7, focally positive for CD10 and negative for Malan A. Overall, the cyst characteristics are those of a type 2 papillary RCC of Fuhrman nuclear grade 3. There was no tumor involvement of the renal capsule or perinephric fat, no vascular space invasion was identified and the margins of resection were free of tumor. The patient was discharged after an uneventful post-operative course. Follow-up control 6 months later showed normal values in the urine analysis and renal function.

\section{Discussion}

Papillary RCC is the second most frequent carcinoma of the proximal renal tubules $(10-15 \%$ of the cases) $(5,6)$. The clinical and histological details were first described by Mancilla-Jiménez et al (7) in 1976. Commonly, it is diagnosed histologically and based on its characteristic enhanced pattern on CT and MRI. Certain characteristics of a cystic renal mass including the character of the lesion wall, septation, calcification, nodularity, CT attenuation values and enhancement are suggestive of a particular diagnosis. However, there is a continuum of radiologic findings often rendering the confident labeling of a cystic renal lesion benign or malignant difficult.

The papillary RCC in the present case was initially characterized by a single cyst with an internal cavity and a calcification filling as part of the cyst. The $1.8-\mathrm{cm}$ diameter distinct cavity was measured using a CT value between -600 and $-970 \mathrm{HU}$, potentially appreciated as air density. To the best of our knowledge, the presence of a necrotic cavity within a papillary RCC has rarely been described. This finding may be considered of interest with respect to the aetiology of the cavity within the tumor mass. We first hypothesized that the intracystic vacuum phenomenon is simply a result of the migration of a gastrointestinal gaseous collection through disrupted renal parenchyma or a renal cyst with inflammatory changes. However, the patient did not experience any fever or abdominal discomfort during the past 22 months. Additionally, the intact renal capsule of the renal specimen and evidence of non-infection of perinephric capsule thickening allows us to exclude the aetiology of gas deposits or inflammatory changes. At histological analysis, infiltration of foamy macrophages into the papillary structures and extensive necrotic tissue with abundant hemosiderin granules suggest a predisposition for degenerative cyst formation.

Extensive necrosis and bleeding within a tumor is often thought to reflect poor tumor vascularization, a characteristic well recognized in angiographic studies of papillary RCC. The comparison between the CT scan in January, 2010 and 22 months later documented the marked progress of the necrotic cavitation of the papillary RCC mass.

\section{Acknowledgements}

The authors thank Dr Kai Ye for his valuable technical assistance during the immunohistochemical analysis.

\section{References}

1. Reuter VE: The pathology of renal epithelial neoplasms. Semin Oncol 33: 534-543, 2006.

2. Presti JC Jr, Rao PH, Chen Q, Reuter VE, Li FP, Fair WR and Jhanwar SC: Histopathological, cytogenetic, and molecular characterization of renal cortical tumors. Cancer Res 51: 1544-1552, 1991.

3. Kovacs G, Wilkens L, Papp T and de Riese W: Differentiation between papillary and nonpapillary renal cell carcinomas by DNA analysis. J Natl Cancer Inst 81: 527-530, 1989.

4. Yamada T, Endo M, Tsuboi M, Matsuhashi T, Takase K, Higano S and Takahashi S: Differentiation of pathologic subtypes of papillary renal cell carcinoma on CT. AJR Am J Roentgenol 191: 1559-1563, 2008.

5. Wein AJ, Kavoussi LR, Novick AC, Partin AW and Peters CA (eds): In: Campbell-Walsh Urology. 10th edition. WB Saunders Company, Philadelphia PA, pp1432-1434, 2012.

6. Störkel S, Eble JN, Adlakha K, Amin M, Blute ML, Bostwick DG, Darson M, Delahunt B and Iczkowski K: Classification of renal cell carcinoma: Workgroup No.1. Union Internationale Contre le Cancer (UICC) and the American Joint Committee on Cancer (AJCC). Cancer 80: 987-989, 1997.

7. Mancilla-Jiménez R, Stanley RJ and Blath RA: Papillary renal cell carcinoma: a clinical, radiologic, and pathologic study of 34 cases. Cancer 38: 2469-2480, 1976. 